\title{
Barriers and facilitators to accessing skilled birth attendants in Afar region, Ethiopia
}

\section{Introduction}

Ethiopia’s Maternal Mortality Ratio (MMR) is one of the highest in Africa, estimated at 676 per 100,000 live births. Maternal deaths account for 30\% of all deaths to women aged 15-49 years (CSA [Ethiopia] and ICF International, 2012). Despite a strong commitment to improving maternal health and reducing maternal mortality, the proportion of women accessing a skilled birth attendant (SBA) is very low. In pastoralist areas, it is even lower. In Afar Region in Ethiopia's north-east, only 7\% of women give birth with a SBA compared to the national average of 15\% (Central Statistical Authority (CSA) [Ethiopia], 2014).

It is extremely difficult to provide accurate maternal and newborn mortality data in many countries, such as Ethiopia. For this reason the proxy measure of the MMR is the number of women birthing with a SBA (doctor, nurse or midwife), ideally in an enabling environment, usually defined as a hospital with equipment, SBAs and supplies to manage obstetric emergencies (Dietsch and Mulimbalimba, 2011). This proxy measure also has difficulties in that by definition, a SBA is considered skilled, no matter what level of expertise and experience they may have. Other caregivers, including traditional birth attendants (TBAs) are by definition, unskilled regardless of their experience, expertise and skills. Furthermore, there is a wide variation in Emergency Obstetric and Newborn Care (EmONC) services provided between and within the different levels of institutional health environments in Ethiopia, including in Afar Region.

Pastoralist areas including Afar Region have poor infrastructure, transportation deficits, harsh environmental conditions and significant inequity in health service utilisation between mobile pastoralists and settled communities. Health Extension Workers (HEWs) are employed in rural areas to refer birthing women to health centres that have limited capacity to provide EmONC. HEWs are generally women with year 10 secondary education and a further 12 months training. They work at the community level and provide an opportunity to reduce the first delay, by facilitating birth preparedness and complication readiness; and mobilise communities to facilitate timely referral to mid-level service providers in health centres (Federal Ministry of Health (FMOH) 2010, 2012). Because of difficulties recruiting enough local pastoralist women, men were permitted to train in Afar Region. 
Afar women have unique needs based upon their cultural and geographical context. This paper explores the barriers and facilitators to accessing SBAs in Afar Region, Ethiopia. The Afar are a large cross border nomadic pastoralist group in the great East Africa Rift Valley. Afar Region, in north-east Ethiopia, is one of the nine autonomous regional states of Ethiopia. Most of the region is dry, rocky and unsuitable for cultivation. The clan, a group of extended families, is the most important political and social unit in Afar culture (Berhe and Adaye, 2010).

In Ethiopia, women are considerably more disadvantaged in terms of literacy, educational attainment, access to paid work opportunities and age at marriage. In Afar Region, levels of literacy, education and outside employment are particularly low: an estimated 75\% of Afar women and $49 \%$ of men have no education, with only $0.5 \%$ women and $2.2 \%$ of men completing secondary schooling. Both government and non-government agencies (NGOs) promote opportunities for Afar adults and children to engage in education (CSA [Ethiopia] and ICF International 2012; Afar Pastoral Development Association, 2014).One of the consequence of women's low education attainment is that the majority of SBAs (nursing and midwifery) workforce is male (Spangler, Barry and Sibley, 2014).

Ethiopia maintains a strong commitment to improving maternal and child health. However, poorly trained staff, cost and quality of services and lack of skilled caregivers all act as barriers (Pitchforth et al., 2010; Fullerton et al., 2013). Whilst the midwifery curriculum in Ethiopia has been developed by education experts, but there has been little input from midwifery educators. While the theory/practice ratio meets criteria set by a number of Ethiopian Ministries, the curriculum is not linked to identified outcomes and individual schools are not held accountable for meeting the standards set (Fullerton et al., 2013).

There is also a critical shortage of midwives, with the FMOH estimating a shortfall of midwives by as much as 95\% (UNFPA, 2011). Ethiopia has a serious deficit of essential services to provide EmONC. Table 1 outlines the levels of maternity service provision in Ethiopia. 
Table 1. Levels of maternity provision in Ethiopia

\begin{tabular}{|l|l|l|}
\hline Maternity Care provider & Facility & Population served \\
\hline $\begin{array}{l}\text { SBAs who are medical } \\
\text { practitioners, midwives and } \\
\text { nurses. }\end{array}$ & $\begin{array}{l}\text { Specialist Hospital } \\
\text { (Tertiary health care) }\end{array}$ & $3.5-5$ million \\
\hline SBA & $\begin{array}{l}\text { General Hospital } \\
\text { (Secondary health care) }\end{array}$ & $\begin{array}{l}1-1.5 \text { million } \\
\text { (Regional) }\end{array}$ \\
\hline $\begin{array}{l}\text { SBA (supported by Health } \\
\text { Officers who are being trained in } \\
\text { surgical procedures such as } \\
\text { Caesareans). }\end{array}$ & $\begin{array}{l}\text { Primary Hospital (Primary } \\
\text { health care) }\end{array}$ & $\begin{array}{l}50-100,000 \text { (Woreda / } \\
\text { Zonal) }\end{array}$ \\
\hline $\begin{array}{l}\text { Mixed (Limited SBA supported } \\
\text { by Health Officers) }\end{array}$ & $\begin{array}{l}\text { Health Centre (Primary } \\
\text { health care with an } \\
\text { average of five satellite } \\
\text { health posts) }\end{array}$ & $\begin{array}{l}15,000-25,000 \\
\text { (Woreda / District) }\end{array}$ \\
\hline $\begin{array}{l}\text { HEWs who are not classified as } \\
\text { SBAs and are supported by } \\
\text { workers in the Health } \\
\text { Development Army (HDA). }\end{array}$ & $\begin{array}{l}\text { Health Post (primary } \\
\text { health care) }\end{array}$ & $\begin{array}{l}3,000-5,000 \text { (Kebele / } \\
\text { village) }\end{array}$ \\
\hline $\begin{array}{l}\text { Traditional Birth Attendants } \\
\text { (TBAs), relatives or unassisted. }\end{array}$ & $\begin{array}{l}\text { Home (illegal in Ethiopia } \\
\text { but the birthplace of } \\
\text { choice for Afar women) }\end{array}$ & N/A \\
\hline
\end{tabular}

Source: This table has been informed by field notes taken on location and by Jackson, (2010), FMOH, (2012), Stover et al., (2014) and Dynes et al., (2014).

The 2014 Mini DHS shows that $45 \%$ of Ethiopian women did not give birth in a health facility because they did not think it was necessary, 33\% of mothers stated that it was not customary, the health facility was too far or that they did not have transportation (22\% of births) (CSA, 2014).

Skilled birth attendance has been identified as the key indicator to track progress towards reducing maternal morality and morbidity. In view of the concerns about underutilisation of delivery and postnatal services, it is important to improve knowledge of factors that may constitute impediments to service use and factors that may facilitate access to services. In Ethiopia, particularly in pastoralist communities, barriers to skilled birth attendance are not well understood, nor are factors that facilitate their utilisation. 


\section{Methods}

Methodology: The project was framed by Key Informant Research (KIR) and Participatory Ethnographic Evaluation and Research (PEER) (Price and Hawkins 2005). The KIR methodology was adapted to facilitate authentic engagement with Afar communities whereby the support of trusted insiders or informants, are used to act as researchers. Informants undertake a brief training program and subsequently identify other members of the community who are suitable interviewees (Price and Hawkins 2005). This methodology is particularly useful when working with disempowered or vulnerable groups, where access by outsiders to sensitive information about sexuality, relationships and reproductive health can be difficult (Price and Pokharel, 2005).

Setting: Data collection was conducted in five districts (woredas) in March 2014 in Afar Region, Ethiopia: Adaar, Dubti, Chifra, Ewa and Kori. The woredas were selected with the support of the Afar Regional Health Bureau (RHB) to provide a cross section of the community. Some woredas have a relatively high population density with greater access to SBA and others are more remote with less access to SBA.

Sample: The researchers worked with the Afar RHB and an Afar NGO, Afar Pastoralist Development Association (APDA), to arrange contacts with health workers and to recruit participants. The 55 participants included eight government and NGO health workers, 14 HEWs and 33 women from the community. Of the 33 women living in the community, most were aged in their 20s (ages ranged from 17 to 49). All but three women were married, only four women were literate and all the women were Moslem. Most women were multiparous and all but four women had experienced the death of one or two children. Most women had attended antenatal care at least once.

Selection and training of HEWs as research team members: With the support of the Afar RHB, HEWs were invited to become research team members. HEWs participated in a threeday workshop where they were trained in ethical research processes and techniques for interviewing community members. During the workshop, HEWs contributed to the development of interview questions and an interview schedule. Ethical considerations were given priority in the workshop, including the ethical recruitment of participants, collection of data and conduct of data analysis. As part of the workshop, HEWs explored how, while 
working with other members of the research team, they would identify key findings emerging from the data.

Lessons the HEWs learned in the research workshop were integrated into their research practice for this project and will be valuable for their own future evaluation skills. Ongoing support and mentoring of the HEWs following the workshop and throughout the data collection and analysis phases of the project were integral to the study design.

There are acknowledged limitations in having the HEWs as researchers including the potential for bias in the ways that participant responses are collected, analysed and reported. There is also the possibility that some participants may not feel comfortable sharing honestly with a researcher with whom there is the potential for a dependent relationship. Much time and discussion was given to the possibility of participant and researcher bias in the three day research training workshop for the HEWS in the section committed to ethical considerations. The HEWs were valued members of a research team who worked with them to identify and overcome potential biases. Furthermore, the advantages of using the HEWs as trusted insiders, fluent in the participant languages and aware of potential research biases outweighed the aforementioned disadvantages.

Data collection: The project utilised a triangulated research design using questionnaires to collect basic demographic data from health workers, HEWs and women in the community. Data were collected during semi-structured interviews and focus groups. Semi-structured interviews were conducted with health workers, HEWs and women. Focus groups were conducted with health workers and HEW and provided more in depth data. Data were collected in Amharic, the national Ethiopian language, as all participants are familiar with the spoken and written language. We worked with interpreters to facilitate data collection from questionnaires, interviews and focus groups.

Data analysis: There were two stages to data analysis. First, the research team members met with HEWs, who had collected the data, and retrieved their interview data during focus groups. This is when HEWs had an opportunity to identify key issues they saw emerging from the interviews. Second, key themes were identified by the research team and the data were analysed based on the pre-existing analytical framework and the research questions that 
were agreed on with the HEWs during the workshop. Thematic analysis of the interviews was in keeping with strategies designed by Annells and Whitehead (2008).

Ethical consideration was given to the utilisation of HEWs as members of the research team and the above mentioned workshop focused on the conduct of ethical research. All participants were provided with a plain language statement and a consent form. Signed consent indicated their willingness to participate in the research program. Participants had the opportunity to withdraw from the interview process at any time, but were advised that once the results were de-identified and amalgamated these results could not be withdrawn.

Ethics approval for the project was gained from Deakin University, Human Ethics Committee, Victoria, Australia (2013-055) and the Federal Democratic Republic of Ethiopia, Federal Ministry of Science and Technology National Research Ethics Review Committee (Phase 27 No 189), Addis Ababa, Ethiopia.

\section{Findings}

The findings are from data collected during HEW interviews with women in the community (CI); interviews with individual health workers (HW); as well as focus groups conducted with HEWs (HEWFG) and Women Health Workers (WEWFG).The findings are grouped into two main clusters. These are: i) facilitators to accessing SBAs; ii) barriers to accessing SBAs current cultural preferences and attitudes about childbirth. Facilitators to accessing SBAs included free ambulance, women's esteem for HEWs, and perceived good quality maternity services at the Health Centres.

HEWs reported that free ambulance transfer to health facilities was improving the timeliness of referrals. In addition, maternity services at a health facility centre or hospital are provided free. These initiatives have improved women’s capacity to access health services,

In the past we believe everything had a charge, but now we realise that health care for pregnant women is free, things are good (HEWFG3)

In keeping with the FMOH (2012) initiatives, the HEWs are involved with improving the uptake of key Maternal, Newborn and Child Health (MNCH) services and disseminating information to create demand and awareness for health services. Participants demonstrated 
positive attitudes towards HEWs and the information and education they brought to the community,

Still the community has a positive attitude to HEWs, in daily activity as well the HEW. They come to our homes, they give education, after we come to the health post if there is a problem. They are willing to do work, they are visiting the pregnant woman at home. They manage a problem they give advice to come to Health Post for follow up (CI, Kori Woreda).

The HEWs have worked to improve knowledge and attitudes to accessing health care facilities and are an integral referral link between the community and health facilities. Some women commented that health services provided comfortable facilities. If the facilities are comfortable and the women are treated well the women will be more motivated to attend,

In our kebele, a pregnant woman was in hospital after she gave birth. They gave her an injection, they gave the child clothes, at that time every facility was given to her. At time of giving birth, they cleaned the blood and took her to another room, all in a good way (WEWFG1).

There is a big difference between having a baby at home and hospital. In the hospital when a woman gives birth, the blood is cleaned. In the house, this is very difficult to clean. In the hospital there is a bed, there is soap, there is water. Before the women were afraid but now, they like the hospital (CI, Dubte Woreda).

Women also had positive comments about some health care providers and attitudes that encouraged further attendance,

When going to the health facility, if the provider is accepting, with a good approach to that woman, she will repeatedly go to that health facility (CI, Elwuha Woreda).

Barriers were identified by women and health workers that hindered their access to a SBA. The most significant barriers to accessing SBA were identified as women's inferior status and limited decision making opportunities as well as perception of poor quality SBA care. Many 
men and women were reluctant to attend health facilities for birthing and commented upon poor service, unfriendly and even abusive SBA treatment. Other identified barriers included inaccessible transportation, long distances on inaccessible or no roads, costs incurred in travelling, purchasing medical supplies and drugs, and competing demands of domestic workloads.

Afar women do not have the power to make their own decisions about accessing health care facilities. There was universal agreement from women in the community that it was appropriate that the men have the power to make decisions. However, this meant that sometimes women were not given permission to seek health care,

The culture gives to the man, everything is decided by his understanding and beliefs, she follows his decisions...The decision maker is only the husband, the female cannot participate in decision making (CI, Elwuha Woreda).

A number of HEWs and women commented upon the capacity of health centre and hospital staff, in terms of skills and attitudes. Some health workers identified gaps in graduating midwife's and nurse's skills,

My observation of the training is that we get graduate midwives and nurses who can tell you the theory but do not appear to be able to apply it to practice. We have some new midwives, and they do have very limited hands on experience. They don't know how to place their hands; they need to be taught the normal mechanisms of birth. But they can tell you everything (HW9).

Women commented upon their perceptions of treatment from of health centre and hospital staff.

Even though we try to talk to health institution staff we have no proper response... they say go to the card room, even though it is an emergency, they say go to the card room. They do not consider how far we are coming; we do not get a service, even though we consider it an emergency, and so we have a negative attitude (CI, Chiffra Woreda). 
The women talk about the nurse, their behaviours, they believe they are unethical. They insult them during delivery, this makes for poor utilisation of health facilities (HEWFG1).

Women also commented upon receiving repeated examinations by many different staff,

Many different nurses/midwives do vaginal examinations, and many staff are putting their fingers into our genitals. After the education we had from the HEWs we are trying to stop everyone touching our genital area. We know from HEWs that they should be checking only every four hours. We are angry with the health facility so we go home and are attended by a TBA selected from the family (CI, Chiffra Woreda).

Many participants, including health professionals and HEWs commented on the lack of resources and SBAs available at the health centres and hospitals. There was consistent commentary about poor equipment, with no water, no electricity and poor sanitation,

They face other problems when they get to the health services - no water, no electricity, no midwife or resources (HW4).

A number of participants explained that they thought they needed to access better services outside their region,

Almost all the women prefer to go out of the region. They assume lack of good instruments, these basic and mandatory instruments, basic vital things they assume they are not here in their health facilities; rather they must use other facilities. There is a lack of medicines, lack of availability and they assume the pharmacy is empty, this means they must go to private institution and incur extra costs for the woman (CI, Chiffra Woreda).

For many Afar women there are great distances to walk before they reach a road which is usually in a poor condition. Many women will travel on foot, or if unable to walk, be carried on a camel or a stretcher, a motor bike or a bajaj (a motor bike with cabin used for public transport), 
It may be that the health facility is a long way from the people. They must follow the water and grass. The community must move and the health facility stays in the same place, sometimes the community cannot get to a health facility (HEWFG5).

Often women attending a health facility had to purchase their own drugs and equipment,

I had to purchase IV fluids and antibiotics from a private pharmacy. There was no water; the toilets were very dirty (HW8).

I know to bring my family to health facility when my family is sick, but after going to health facility it costs more money, so these things are difficult (CI, Dubti Woreda).

There are other costs incurred in accessing services: any men carrying the woman and her accompanying family have to be fed and accommodated.

Afar men are acknowledged as the decision makers and sometimes this means women cannot get permission to travel to health care facilities. The women's work is commonly given higher priority than access to health care.

Afar women are responsible for caring and protecting their children and livestock (goats, cattle, sheep and camels). They carry water, prepare food and complete other domestic duties. In the dry season, the women may walk more than eight hours per day to get water. They are frequently malnourished and malaria, anaemia and hookworm infestations are common but the needs of others this must take priority over her own needs,

The woman supports the family by going to the cattle, rather than giving priority to herself...if no water is available, even if she is sick, her priority is to get water for her family (CI, Kori Woreda).

Current cultural preferences and attitudes about childbirth play a significant role in whether or not women access SBA. There were many comments about the negative behaviour of SBAs and many of these comments reflect the unprofessional, culturally unsafe practices that offend Afar men and women. 
Women expressed their preference to birth at home with a TBA. Afar women are shy and secretive about pregnancy and birthing. Commonly their pregnancy is hidden and they prefer to give birth quietly at home. It is traditionally women's business; neither men nor women want the woman to be attended by a male SBA,

The men cannot accept that the woman will expose herself to other men (HEWFG5).

The TBA continues to be the preferred caregiver and TBAs still attend the majority of birthing women in Afar,

The TBAs are preferred, the women cannot go to the health facility because they will cut an episiotomy and the male will see. It is better to protect women's privacy and stay at home. The TBAs are confidential, they keep the privacy, keep the blood from the husband (HEWFG1).

Of course, we use the TBA, in ancient times it has come as our culture, this is the only option in the past. The TBA is our relative, she is like our family (CI, Chifra Woreda).

TBAs are not only an integral part of Afar culture, they are admired and respected elder women who are completely accepted by the community, to the extent, that even though female, they are accepted as decision makers,

The TBA is admirable; she is strongly working without complaint. She protects the woman and is effective. The TBA saves the time and money of the women and community. The community is admiring of this woman (CI, Chiffra Woreda).

We believe the TBA is a woman who is a decision maker...If there is bleeding before delivery, if also the baby is breech or it is difficult, the TBA will decide to bring the woman to the Health Centre. The community will accept this and bring the woman to the Health Centre. She has the power to decide to bring the woman to the Health Centre (CI, Kori Woreda).

The TBA is experienced, available and can care for the woman throughout the labour and birth, 
Of course the woman use TBAs, they are in proximity, living in the community. The TBA can get there early, they are experienced, they have attended many childbirths. The community believe in this woman because she has experience. Also the TBA attends the woman in her house, she does not have to expose herself to others, her security and privacy is protected (CI, Elwuha Woreda).

If the TBA decides that the woman must go to a health care facility then the men and women will accept this decision. The participants often compared and contrasted role of the TBA with that of the SBA,

They believe the TBA will make a secret of everything, they believe the nurse will expose the woman. The TBA is there starting from the labour to delivery in case of a health professional they do not do this. They do not attend for the whole labour and birth (CI, Kori Woreda).

The participant's perception was that the TBAs afforded safer, more culturally appropriate care than the SBA and hence the TBA was the caregiver of choice for most Afar women.

\section{Discussion}

Findings from this research identify reduced costs; HEW's integral referral link between community and hospital facilities, comfortable facilities and respectful care all act as facilitators to women's access to SBA. The barriers to access include women's low status and lack of decision making, perceptions of poor quality of care, unfriendly, even abusive treatment by SBA, prevalence of culturally inappropriate male SBA, prohibitive distances and costs associated with accessing services. Furthermore, there is a strong cultural preference for Afar women to birth at home, supported by a TBA. The Ethiopian Government's promotion of SBAs and institutional birth reflects the global, systemic approach to reduce maternal and newborn mortality but this may threaten Afar women's cultural safety. The researchers acknowledge the challenges the Ethiopian Government faces in their endeavours to provide Afar women with access to culturally safe and acceptable skilled birth attendance in an enabling environment. Afar men and women trust and rely heavily upon TBAs despite policy interventions that have shifted from recognition of the 
TBA's longstanding role in assisting women to birth (Tesfaye, 2010; Warren, 2010) to zero tolerance of women birthing at home with a TBA in Ethiopia (Koblinsky, 2014).

The Ethiopian government is promoting referral linkages between the community and health facilities through HEW referrals, provision of free ambulances, and improving basic emergency obstetric and newborn care in primary health facilities (Fesseha et al., 2013). However, when an Afar woman needs or chooses to access a SBA, they face unique challenges. Afar men and women believe it is better to birth at home, attended by a trusted and respected TBA who will maintain the privacy, integrity, security and safety of the woman. Research conducted in Afar Region found that over 90 percent of women were attended by TBAs in Zone 3 of Afar Region during the birth of their last child at home (Yousuf et al., 2010). There was also a strong preference for home birth linked to childbirth practices such as birthing in a sitting position, which was not available in a medical facility, and being able to perform rituals and festivities upon the successful birth of a healthy child (Yousuf et al., 2011).

A number of studies identify gaps in the facilitation of culturally sensitive care during childbirth; including recognition of important traditions (Warren, 2010). Research conducted in rural Southern Ethiopia found that while many women attended antenatal care, $86 \%$ of women birthed at home with a TBA. The main reasons were because women did not believe it necessary or customary to use a SBA, and the TBA was perceived as the more culturally acceptable and competent health worker. In comparison, the community women had a low opinion of the competency and availability of the SBA who did not allow for cultural practices such as taking the placenta home for burial. Furthermore, there was no provision for privacy and confidentiality in the health centre (Shiferaw et al., 2013).

Evidence shows that disrespect and abusive care at birth acts as a powerful deterrent to SBA care and contributes to significant suffering and human rights violations of women (Bowser and Hill, 2010). The White Ribbon Alliance Charter for Respectful Maternity Care (2011) recognises that women in many countries do not receive respectful maternity care (UNFPA, 2014). Examples of disrespect and abuse of women include: verbal and physical abuse, insulting women, non-consented care, and discrimination based upon specific physical or ethnic attributes and lack of confidentiality (Bowser and Hill, 2010). Features that contribute to the incidence of disrespect and abuse of women during childbirth are multifactorial. They 
include a normalisation of disrespect and abuse during childbirth, lack of community engagement of professionals, lack of professional autonomy and empowerment. Other factors relating to national and professional governance of the professions, deficiencies in health services and human resources also contribute to the problem (Bowser and Hill, 2010).

There is evidence that integration of TBAs with formal health systems can increase access to SBA and reciprocal learning between caregivers (Byne and Morgan, 2011; Dynes et al., 2014). Greater mutually respectful collaboration could be promoted at national, regional and local levels. The TBA is the preferred choice and sometimes the only option for many Afar women and this is unlikely to change in the short term future. Other Ethiopian studies have commented on a need to strengthen collaboration and community referral pathways between HEWs, community health workers and TBAs (Dynes et al., 2014; Shiferaw et al., 2013; Pitchforth et al., 2010; Warren, 2010;). A 2010 scoping exercise recommended greater collaboration of HEWs with other health providers, community health workers and TBAs to ensure continuity of care, birth preparedness, early recognition of complications and social support for childbearing women (Tesfaye, 2010). TBAs are a key variable in the outcome of childbirth whereby they influence the birth preparedness, the progress and hygiene of childbirth, and the recognition and referral of women experiencing complications (Warren, 2010).

There is a common perception that the health facilities are poorly resourced and staff may be unfriendly, unprofessional or unavailable (Gobezayehu et al., 2014; Shiferaw et al., 2013; Fullerton et al., 2013; Warren, 2013). The Ethiopian government recognises that the relationship between the health sector and the community must be improved. Health Sector Development Program (HSDP) IV specifically notes that strategies are needed to develop and deepen the health sector's relationship with the community. The 'Customer value proposition of HSDP-IV' outlines principles which should underpin its relationship with the community. The health services should aim to portray characteristics of trustworthiness, professionalism, customer friendliness, building respectful, harmonious and equitable relationships with women. Poor birthing room environment and poor attitude of SBAs was identified as a challenge (FMOH, 2010).

\section{Implications for midwifery practice:}


The Afar community are observant and responsive to facilities and health care providers who they perceive as respectful and safe: in contrast, disrespectful or abusive treatment and inadequate facilities act as a serious disincentive to access to SBA (Shiferaw et al., 2013; Warren, 2013; Fullerton et al., 2013; Bower and Hill, 2010). The global community is recognising the deleterious impact of an entrenched culture of abuse and disrespectful treatment during childbirth. Ethiopian midwives are predominately male but Afar men and women find female birth attendants more culturally acceptable. An understanding of what women want and need during the childbirth continuum, and the provision of respectful, woman centred care is the starting point for developing quality in maternity services (Freedman and Kruk, 2014). National and local health authorities and midwifery professional governance should no longer tolerate disrespectful and abusive treatment of women during childbirth.

In Ethiopia, provision of culturally safe care will improve the utilisation of SBAs. Priority can be given to enhancing the attitudes and skills of SBAs, and through provision of medical equipment and supplies to ensure that women have access to respectful, well trained and well-resourced SBAs. Respectful woman-centred care can be integrated into curriculum for midwives and other SBAs. These principles can be reinforced through national professional and health service governance structures.

Women comprise a small percentage of the maternity health care workforce. The Ethiopian government can promote education for girls and women thorough positive discrimination and incentives to complete schooling and undertake study in midwifery and other health care disciplines. At the time of writing there were no Afar students enrolled in the Midwifery program in Semera University, Afar Region.

\section{Limitations}

Interviews and data collection from the community conducted by HEWs may have inhibited the responses of the interviewees, in particular their freedom of expression in the context of commentary upon the role of HEW in the community. Understanding in the interview processes and then the subsequent feedback provided by HEWs as KIR to the principal researchers may have been impacted by the need to use interpreters, the quality of translation 
and comprehension of the HEWs. The research was conducted in Afar Region and findings are not generalisable to any other population group.

\section{Conclusion}

Nomadic Afar women experience unique difficulties in accessing health care and SBAs. The Ethiopian government and all cadres of health workers have profound challenges in delivering adequate health care to this region. Midwives and other health workers work in extreme conditions to provide care with minimal or non-existent supplies and facilities. The maternity workforce may be improved with further capacity building and resourcing. Curriculum development, enhanced government regulation and governance of the midwifery profession may contribute to this capacity building. Ongoing mentoring and leadership through the Ethiopian Midwives Association could promote professional culturally safe and competent midwifery care. Midwifery academics should integrate components of cultural security, professional behaviours and attitudes into midwifery curricula. Afar women would benefit from strengthened, respectful collaboration between community-based TBAs and SBAs within mainstream health services.

\section{References}

Afar Pastoral Development Association 2014. Retrieved from http://www.apdaethiopia.org/education.html

Annells, M., Whitehead, D., 2008. Analysis data in qualitative research. In Schneider, Z., Whitehead, D., Elliott, D., Lobiondo-Wood, G., Haber, J., (Eds.), Nursing and Midwifery Research: Methods for Appraisal for Evidence Based Practice. Elsevier. Chatswood NSW, pp. 138-154.

Berhe, T., Adaye, Y., 2010. Researching local conflicts and regional security. Institute for Security Studies. Pretoria, South Africa.

Bower, D., Hill, K., 2010. Exploring Evidence for Disrespect and Abuse in Facility-Based Childbirth. A Report of a Landscape Analysis. USAID-Traction Project. Harvard School of Public Health.

Byne, A., Morgan, A., 2011. How the integration of traditional birth attendants with formal health systems can increase skilled birth attendance. International Journal of Gynaecology and Obstetrics Vol 115(2). 127-134. DOI: 10.1016/j.ijgo.2011.06.019.

Central Statistical Authority [Ethiopia], 2014. Ethiopia Mini Demographic and Health Survey 2014, Addis Ababa. 
Central Statistical Authority [Ethiopia] and ICF International, 2012. Ethiopia Demographic and Health Survey 2011, Addis Ababa, Ethiopia and Calverton, Maryland, USA

Dietsch, E. Mulimbalimba, L., 2011. Reconsidering the value of traditional birth attendants: a literature review. MIDIRS: Midwifery Digest, 21(1): 119-124 reprinted in full with permission from African Journal of Midwifery and Women’s Health, 4(3):133-138.

Dynes, M., Stephenson, R., Hadley, C., Sibley, L., 2014. Factors shaping interactions among community health workers in rural Ethiopia: Rethinking workplace trust and teamwork. Journal of Midwifery and Women’s Health 59. S32-S43.

DOI:10.1111/jmwh.12135.

Federal Ministry of Health (FMOH). 2012. Road Map for Accelerating the reduction of Maternal and Newborn Morbidity and Mortality 2012-2015. Addis Ababa.

Federal Ministry of Health (FMOH). 2010. Health Sector Development Plan (HSDP) IV 2010/11-2014/15. Retrieved from

http://www.nationalplanningcycles.org/sites/default/files/country_docs/Ethiopia/ethio pia_hsdp_iv_final_draft_2010_2015.pdf

Fesseha, N., Karim, A.M., Betemariam, W., Gebrekiristos, T., 2013. Implementing innovative solutions for effective referral for critical maternal and newborn health conditions in Ethiopia. American Public Health Association On line Program http://l10k.jsi.com/Resources/Docs/Referral_APHA2013.pdf

Freedman, L., Kruk, M., 2014. Disrespect and Abuse of Women in Childbirth: challenging the global quality and accountability agendas. Lancet Published Online June 23, 2014 http://dx.doi.org/10.1016/ S0140-6736(14)60859-X

Fullerton, T., Johnson, J., Thompson, J., Vivio, D., 2013. Quality considerations in midwifery pre-service education. Exemplars from Africa Midwifery 29(10).1129-1136

Gobezayehu, A.G., Mohammed, H., Dynes, M., Desta, B.F., Barry, D., Aklilu, Y., 2014. Knowledge and Skills Retention Among Frontline Health Workers: Community Maternal and Newborn Health Training in Rural Ethiopia'. Journal of Midwifery and Women's Health 59(s1). S21-S31.

Jackson, R., 2010. (Un)safe routes: Maternal mortality and Ethiopia's development agenda, Unpublished thesis, Deakin University.

Koblinsky, M., 2014. Reducing Maternal and Perinatal Mortality through a Community Collaborative Approach: Introduction to a Special issue on the Maternal and Newborn Health in Ethiopia Partnership (MaNHEP). Journal of Midwifery and Women's Health 59(1).S1-S5.

Pitchforth, E., Lilford, R., Kebede, Y., Asres, G., Stanford, C., Frost, J., 2010. Assessing and understanding quality of care in a labour ward: A pilot study combining clinical and social science perspectives in Gondar. Ethiopia. Social Science and Medicine, doi: 10.1016/j.socscimed.2010.08.001. 
Price, N., Hawkins, K., 2005. The peer ethnographic method for health research: methodological and theoretical reflections. In Holland, J., Campbell, J., (Eds.), Methods in Development Research: Combining Qualitative and Quantitative Approaches, Intermediate Technology Publications Ltd, Warwickshire. pp. 149-161.

Price, N., Pokharel, D., 2005. Using key informant monitoring in safe motherhood programming in Nepal. Development in Practice 15(2). 151-164.

Shiferaw, S., Spigt, M., Godefrooij, M., Melkamu, Y., Tekie, M., 2013. Why do women prefer home births in Ethiopia? BMC Pregnancy and Childbirth 13(5). doi:10.1186/1471-2393-13-5

Spangler, S., Barry, D. Sibley, L., 2014. An evaluation of Equitable Access to a communityBased Maternal and Newborn Health Program in Rural Ethiopia. Journal of Midwifery and Women’s Health 59. S101-S109. DOI: 10.1111/jmwh.12133

Stover, K.E., Tesfaye, S., Frew Hailemichael, A., Mohammed, H., Barry, D., Alamineh, L., Teshome, A., Hepburn, K., Sibley, L. 2014. Building District-Level Capacity for Continuous Improvement in Maternal and Newborn Health. Journal of Midwifery and Women’s Health 59. S91-S100. DOI: 10.1111/jmwh.12164.

Tesfaye, S., 2010. A National Scoping Exercise and Strategic Recommendations for Working with Individuals, Families and Communities to improve Maternal and Neonatal Health in Ethiopia. Ethiopian Journal of Health Development 24. Special Issue 1. 89-91

UNFPA, 2014. The State of the Worlds Midwifery: A Universal Pathway: A Woman's Right to Health. Retrieved from http://unfpa.org/public/home/pid/16021

UNFPA, 2011. The State of the Worlds Midwifery: Delivering Health, Saving Lives. http://www.who.int/pmnch/media/membernews/2011/2011_sowmr_en.pdf

Warren, C., 2010. Care seeking for maternal health: challenges remain for poor women. Ethiopian Journal of Health Development 24. Special Issue 1.100-104

Warren, C., Nuke, R., Abuya, T., Ndwiga, C., Maingi, G., 2013. Study protocol for promoting respectful maternity care initiative to assess, measure and design interventions to reduce disrespect and abuse during childbirth in Kenya. BMC Pregnancy and Childbirth. 13- 21. doi:10.1186/1471-2393-13-21

Yousuf, J., Mulatu, T., Nigatu, T., Seyum, D., 2010. Revisiting the Exclusion of Traditional Birth Attendants from Formal Health Systems in Ethiopia. AMREF Discussion Paper Series.

Yousuf, J., Ayalew, M., Seid, F., 2011. Maternal health beliefs, attitudes and practices among Ethiopian Afar'. Exchange 1. 12-14. 